\title{
Experiments in medical research
}

\section{F Passariello 1}

${ }^{1}$ Fondazione Vasculab ONLUS, via Francesco Cilea 280 - 80127 Naples, Italy

submitted: Dec 31, 2017, accepted: Dec 31, 2017, EPub Ahead of Print: Dec 31, 2017, published: Dec 31, 2017

Conflict of interest: None

DOI: 10.24019/jtavr.38 - Corresponding author: Dr. Fausto Passariello, afunzionale@tiscalinet.it

(C) 2017 Fondazione Vasculab impresa sociale ONLUS. All rights reserved.

Experimental research is generally considered predominant in Medicine, in comparison with other articles regarding theories, reviews, etc..

Medical journals as well as many meeting organizations, nowadays prefer scientific contributes, which fit the pre-requisite of the well-known IMRaD $^{1}$ sequence: introduction, aims, materials and methods, results, discussion and conclusion. This strict scheme is ubiquitary, even if often erroneously applied to all types of medical research ${ }^{2}$.

The current issue of JTAVR guests 2 original articles, which are compliant with this accepted standard scheme, presenting relevant data about compression therapy (Rastel) and venous diagnostics (Ermini).

The paper of Rastel deals with the proposal of a new device, able to measure locally the "ex-vivo" pressure of compression stockings, when donned on the leg. The aim is to replace the time consuming but highly reliable, normalized measurement in a textile laboratory with a less precise hybrid "ex-vivo" method, which is however even more precise than the "in-vivo" measurements, gathered with wearable devices.

The paper of Ermini practically shows how a venous dynamic test, which is daily practised in most vascular labs since more than 20 years, can gain a reliable corroboration, when submitted to validation through a strict experimental protocol against a generally accepted manoeuvre like the squeezing test. In this way, daily practice enters the promising world of scientific validation.

Although there are a lot of useful papers, which explain in clear words the way to successfully write a scientific article, let me remind you all some non-written rules, which can be of paramount importance in setting the value of your manuscript.

Both theoretical and experimental research have their own well-defined specificity. It is therefore essential to set clearly the category of the manuscript, avoiding mixing in the same text a new theory and a new experiment. Practically both deserve a separate article, with a deep description of their details.

An experimental article should describe the theoretical and literature premises in the introduction, justifying the aims of the paper. Practically, from Theory to Experiment.

In the same time, it should show any changes in the theoretical frames in the discussion, what the paper adds to the current literature. Practically, from Experiment to Theory.

Methods and Results should be self-contained, i.e. they should not have direct relationship (apart of references) with the other parts of the paper.

There must be a continuous osmosis between the experimental research and the models and intepretations of literature, even if already accepted or still in evaluation.

An experimental research, which does not refer to literature or to a theoretical frame, is just a blind research, which drives you nowhere.

Finally, an experimental research should suggest how to go on in the neverending process of scientific research.

Following these suggestions, we will find us all together after the same rainbow's end as research friends.

Fausto Passariello

Editor in Chief 
F Passariello - Experiments in medical research

\section{References}

1) International Committee of Medical Journal Editors. Recommendations for the conduct, reporting, editing, and publication of scholarly work in medical journals. http://www.icmje.org/icmje-

2) Theories and experiments in medical research. Passariello F. recommendations.pdf (2016, accessed April 2017). 